\title{
Dual-electrochromic bipolar electrode-based universal platform for the construction of various visual advanced logic devices
}

\author{
Qingfeng Zhai ${ }^{1,2,3}$, Daoqing Fan ${ }^{1,2,3}$, Xiaowei Zhang ${ }^{1,2}$, Jing $\mathrm{Li}^{1,2}$ and Erkang Wang ${ }^{1,2}$
}

A universal logic platform based on dual-electrochromic bipolar electrodes (BPEs) for the operation of various visual advanced logic devices was designed and constructed for the first time. Two BPEs separated by three reaction channels filled with different reaction solutions constituted the closed BPE system and were used as the initial state. Under different input combinations, the electrochemical oxidation of 2, 2' -azinobis (3-ethylbenzthiazoline-6-sulfonic acid) and the electrodeposition of Prussian blue occurred at different poles simultaneously and triggered rapid color changes that could be easily visualized by the naked eye. By defining the color changes at the two specific poles as outputs, visual advanced logic devices, including an encoder, a decoder, a demultiplexer, a keypad lock and a three-input concatenated logic circuit with two outputs, were successfully constructed.

NPG Asia Materials (2017) 9, e421; doi:10.1038/am.2017.132; published online 18 August 2017

\section{INTRODUCTION}

With the burgeoning developments of Boolean logic computation, various unconventional logic systems ${ }^{1-3}$ have been constructed to operate different types of basic, ${ }^{4-6}$ advanced $^{7-9}$ and concatenated molecular logic devices. ${ }^{10-12}$ Although great research achievements have been realized in this area, there is still considerable room for progress. ${ }^{13,14}$ One significant limitation is that most of the previously reported advanced logic gates (such as full/half adder/subtractor, ${ }^{15}$ encoder/decoder, ${ }^{7,16}$ and multiplexer/demultiplexer ${ }^{17,18}$ ) were fabricated using luminescent ${ }^{19,20}$ or traditional electrochemical platforms, ${ }^{10,21}$ whose outputs (luminescence, voltage, current) were often recorded using additional equipment and difficult to visualize by the naked eye. By contrast, colorimetric logic platforms present visual outputs that are rapidly responsive, easy-to-read, and could be observed by the naked eye. To the best of our knowledge, although many basic colorimetric logic gates (for example, AND, ${ }^{22}$ INHIBIT, ${ }^{23,24} \mathrm{OR}^{23}$ ) have been developed, only few single-output colorimetric advanced logic devices have been reported (keypad locks ${ }^{25,26}$ or some simple concatenated logic circuits $^{23,27}$ ). This result is mainly because it is difficult to integrate two separate visible signals, which is necessary for fabricating dual-output advanced logic devices, into one reaction system. To resolve this issue, a universal platform that can operate various visual advanced logic devices with more than one colorimetric output is highly desirable and requires further investigation.

A bipolar electrode (BPE) is an electronic conductor that can act as both an anode and a cathode in solution wirelessly under a power supply. ${ }^{28-30}$ Logic computation based on bipolar electrochemistry has attracted tremendous interest recently owing to its ease of construction, integration and control. ${ }^{25,31-33}$ Crooks pioneered the integration of logic circuits with a split BPE using electrochemiluminescence as an output signal in an open BPE system. ${ }^{33}$ However, the utilization of the open BPE mode limited further applications due to the low current efficiency. Moreover, only basic logic gates were operated in his work. By comparison, closed BPEs with ultrahigh current efficiency and non-interfering reactions at the two poles separated by different reservoirs have become increasingly popular. ${ }^{34-36}$ Liu et al. ${ }^{25}$ fabricated a visual keypad lock based on a closed mode single-electrochromic BPE, but the single-output platform could not efficiently meet the requirements of visual advanced or concatenated logic devices. To overcome these limitations, more than one colorimetric signal should be integrated into one closed BPE system.

Herein, a universal dual-electrochromic logic platform was constructed for the first time by introducing the electrochemical oxidation of 2, 2'-azinobis (3-ethylbenzthiazoline-6-sulfonic acid) (ABTS) and the electrodeposition of Prussian blue (PB) into one closed BPE system. This platform consists of two BPEs separated by three reaction channels in which the color switching of ABTS (no color change/turn green) and PB (no color change/turn blue) at the two poles will not interfere with each other. Based on the smart design of a bipolar electrochemical system, various advanced logic devices whose outputs were recognized via the naked eye, including an encoder, a decoder, a demultiplexer, a keypad lock and a three-input concatenated logic

\footnotetext{
${ }^{1}$ State Key Laboratory of Electroanalytical Chemistry, Changchun Institute of Applied Chemistry, Chinese Academy of Sciences, Changchun, China and ${ }^{2}$ University of Chinese Academy of Sciences, Beijing, China

${ }^{3}$ These authors contributed equally to this work.

Correspondence: J Li or Professor E Wang, State Key Laboratory of Electroanalytical Chemistry, Changchun Institute of Applied Chemistry, Chinese Academy of Sciences, No. 5625, Renmin Street, Changchun, Jilin 130022, China.

E-mail: lijingce@ciac.ac.cn or ekwang@ciac.ac.cn

Received 23 December 2016; revised 24 March 2017; accepted 31 March 2017
} 
Table 1 The comparison results of this work compared with other proposed platform based logic devices

\begin{tabular}{llll}
\hline Platform & Output signal & Operation time & Logic devices \\
\hline GO/DNA-Ag nanocluster & Fluorescence & About $40 \mathrm{mins}$ & Encoder, decoder \\
DNA/Cu nanoparticle & Fluorescence & About $40 \mathrm{mins}$ & Multivalued logic gates \\
DNAzyme & Fluorescence & About $1 \mathrm{~h}$ & Multiplexer, demultiplexer \\
Electrochemical (DNA and redox probe) & Electrochemiluminescence & More than $1 \mathrm{~h}$ & Encoder, decoder \\
Electrochemical (DNA and redox probe) & Current & More than $1 \mathrm{~h}$ & Encoder, decoder \\
BPE & Dual-visual (colorimetric) & About 5 mins & Encoder, decoder, demultiplexer, concatenated logic circuit $\quad$ This work
\end{tabular}

circuit with dual outputs, were constructed easily by modifying the input combinations. This logic platform presented multiple advantages compared with previous ones, as presented in Table 1.

\section{MATERIALS AND METHODS}

\section{Chemicals and reagents}

All chemicals were of at least analytical grade and were used without any further purification. ABTS and glutathione (GSH) were purchased from Sigma Aldrich (Shanghai, China) (http://www.sigmaaldrich.com). Polydimethylsiloxane (PDMS) and curing agent were obtained from GE Toshiba Sillicones Co., Ltd (Shanghai, China). Indium tin oxide (ITO)-coated glass substrate (resistance: $\sim 6 \Omega$ /square) was obtained from CSG Holding Co., ltd. (Shenzhen, China). In this work, $10 \mathrm{~mm}$ phosphate buffer solution with $100 \mathrm{~mm} \mathrm{KCl}$, $\mathrm{pH}=7.4$ was used, and high concentrations of ABTS and $\mathrm{H}_{2} \mathrm{O}_{2}$ solution were freshly prepared before use and then diluted to the final concentration. The PB deposition solution used in this work was composed of $2.5 \mathrm{~mm} \mathrm{FeCl}_{3}+2.5 \mathrm{~mm}$ $\mathrm{K}_{3} \mathrm{Fe}(\mathrm{CN})_{6}+0.1 \mathrm{M} \mathrm{HCl}+0.1 \mathrm{M} \mathrm{KCl}$. The water used in all experiments was purified by a Milli-Q system (Millipore, Bedford, MA, USA).

\section{Device fabrication}

As reported in our previous work, ${ }^{36}$ a standard photolithographic technique was employed to fabricate patterned ITO electrodes. In brief, the clean ITO substrate was spin-coated with RZJ 390 photoresist. The coated ITO substrate was covered by a photomask containing the designed patterns (Supplementary Figure S1A) and exposed to UV irradiation for $9 \mathrm{~s}$. The exposed photoresist layer was removed by $0.1 \mathrm{M} \mathrm{NaOH}$, and the exposed ITO was etched with $\mathrm{HCl}-$ $\mathrm{FeCl}_{3}$ solution. Finally, the patterned ITO electrodes were obtained by removing the remaining photoresist with ethanol, and the closed bipolar system was achieved through the bonding of a polydimethylsiloxane membrane with a reservoir onto the patterned ITO substrate (Supplementary Figure S1B).

\section{UV-vis spectra measurement}

The UV-vis spectra of the color changes of the ABTS and PB on the surface of the electrodes were measured using a UV-vis spectrophotometer. Because the PB was deposited directly on the electrode, the ITO glass was placed in a suitable position in the UV-vis spectrophotometer so that the incident light source passed through pole IV completely (Supplementary Figure S3). The UV-

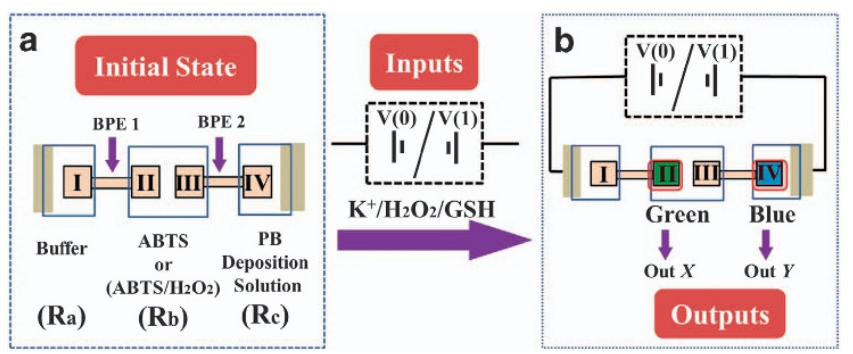

Scheme 1 Structure of the closed BPE system with two BPEs separated by three reaction channels and definition of the initial states of the platform (a). Definition of two visual outputs: Out $X$ (Green, on pole II) and Out $Y$ (Blue, on pole IV) under different inputs (b). vis spectrum of $\mathrm{PB}$ was collected from $450 \mathrm{~nm}$ to $1000 \mathrm{~nm}$. To measure the absorbance of ABTS, the solution on the surface of pole II was collected and transferred to an ultramicro- spectrophotometer/fluorometer (DS-11 FX+, Denovix, Wilmington, DE, USA), and the spectra were collected from $390 \mathrm{~nm}$ to $450 \mathrm{~nm}$.

\section{Operation of the developed advanced logic device}

Before operating different logic computations, the driving voltage for $\mathrm{PB}$ deposition and ABTS electrochemical oxidation were optimized in the absence or presence of $5 \mathrm{mM} \mathrm{H}_{2} \mathrm{O}_{2}$. A CHI 760E electrochemical workstation (Chenhua Instruments Shanghai Co., Ltd, China) was used to supply voltage to the driving electrodes for $180 \mathrm{~s}$. The advanced logic devices, including an encoder, a decoder, a demultiplexer, a keypad lock and a three-input concatenated logic gate, were operated at the optimal voltage. Detailed information is provided in Supporting Information.

\section{RESULTS AND DISCUSSION}

\section{Definition of the initial state and outputs}

As shown in Scheme 1a, the closed BPE system was composed of two BPEs (BPE 1 and BPE 2) separated by three reaction channels, designated $\mathbf{R a}, \mathbf{R b}$ and $\mathbf{R} \mathbf{c}$. Before the operation of different logic devices, phosphate buffer, ABTS/ $\mathrm{H}_{2} \mathrm{O}_{2}$ or ABTS $\left(\mathrm{ABTS} / \mathrm{H}_{2} \mathrm{O}_{2}\right.$ for the encoder and decoder; ABTS for the demultiplexer, keypad lock and concatenated logic circuit) and $\mathrm{PB}$ deposition solution were added to reservoirs $\mathbf{R a}, \mathbf{R b}$ and $\mathbf{R} \mathbf{c}$, respectively. Two BPEs separated by the three reaction channels, which were filled with the different reaction solutions, constituted the closed BPE system, and this system was defined as the initial platform state. To satisfy the requirements of different logic devices, the driving voltage with different directions and suitable values/supply time, $\mathrm{K}^{+}, \mathrm{H}_{2} \mathrm{O}_{2}$ and GSH were alternatively used as the inputs. For the voltage input, the negative voltage (left ' + ', right ' - ', designated as ' $+/-$ ') and positive voltage (left '-', right ' + ', designated as ' $-/+$ ') were defined as ' 0 ' and ' 1 ', respectively. For $\mathrm{K}^{+}$, $\mathrm{H}_{2} \mathrm{O}_{2}$ and $\mathrm{GSH}$, the absence and presence of these inputs were defined as ' 0 ' and ' 1 ', respectively.

Before the definition of two outputs, the introduction of $\mathrm{H}_{2} \mathrm{O}_{2}$ as an input to this system should be explained initially. As reported by many BPE-based electrochromic works and verified in our experimental results (Supplementary Figure S4), both the electrochemical oxidation of ABTS/ABTS. ${ }^{+}$and the electrodeposition of $\mathrm{PB}$ induce color changes that could occur at the extremities of the two BPEs under sufficient driving voltage. ${ }^{37,38}$ If the voltage was applied as the solely input, only single-input triggered logic device can be achieved. To meet the input requirements of different logic devices and make the system a universal platform, in addition to the voltages, one essential reactant should be introduced into the system to regulate the color change reaction. ABTS can be effectively oxidized by $\mathrm{H}_{2} \mathrm{O}_{2}$ and turns green in the typical colorimetric reaction. ${ }^{39}$ In addition, as $\mathrm{H}_{2} \mathrm{O}_{2}$ has a low redox potential, ${ }^{40}$ the supplied driving voltage can also effectively promote the oxidation of ABTS in $\mathbf{R b}$ and the deposition of $\mathrm{PB}$ at the 
BPE cathode in Rc simultaneously owing to the 'connected in series' design of the closed BPE system that can be further confirmed through the current change before and after addition of $\mathrm{H}_{2} \mathrm{O}_{2}$ (Supplementary Figure $\mathrm{S} 2$ ). Thus, $\mathrm{H}_{2} \mathrm{O}_{2}$ was chosen as another input in the platform to regulate the color changes of ABTS (no color change/turn green) and deposited PB (no color change/turn blue) simultaneously. The driving voltages were optimized initially to obtain the highest signal-to-noise ratio of the absorption values of ABTS. ${ }^{+}$and deposited $\mathrm{PB}$ in the presence and absence of $\mathrm{H}_{2} \mathrm{O}_{2}$, as shown in Supplementary Figure S4. A negative voltage of $-4 \mathrm{~V}(3 \mathrm{~min})$ and a positive voltage $+3.8 \mathrm{~V}$ ( $3 \mathrm{~min}$ ) were used in subsequent experiments. The color changes at pole II were taken as Out $\boldsymbol{X}$ (no color change $=$ '0', turn green $\left(\mathrm{ABTS}^{+}\right)=$' 1 '), and those at pole IV were defined as Out $\boldsymbol{Y}$ (no color change $=$ '0', turn blue (deposited PB) $=11$ '), as shown in Scheme $1 \mathrm{~b}$. Thus, the initial, input and output states of the platform were as defined above. The coloring mechanism of $\mathrm{ABTS}^{+}$and deposited PB is shown in Supplementary Scheme S1.

\section{Operation of a 2-to-1 encoder and a 1-to-2 decoder}

An encoder/decoder is a digital device that converts the data/code into code/data through information storage or transmission.7,17,41 The initial state of the encoder and decoder is presented in the center of Figure 1a. To validate the proposed strategy, a single-output 2-to-1 encoder was first designed. The voltage directions were used as one input (IN 1), $\mathrm{K}^{+}$(IN 2) (which has no effect on the system) was used as an additional input, and the color changes at pole IV were defined as the encoder's output (left part of Figure 1a). In the presence of a negative voltage $(+/-)$ and $\mathrm{K}^{+}\left(\mathrm{IN} \mathbf{1}={ }^{\prime} 0\right.$ ', IN $2={ }^{\prime} 1$ '), PB will be deposited on the right driving cathode and not on the anode of BPE 2 (pole IV), and no color change was observed on pole IV, yielding the output ' 0 ', as shown in Figure 1a (a). In the presence of a positive voltage $(-/+)$ and the absence of $\mathrm{K}^{+}\left(\mathrm{IN} \mathbf{1}=11\right.$ ', IN $2={ }^{\prime} 0$ '), the polarity of the two BPEs was reversed accordingly. $\mathrm{PB}$ was deposited on the cathode of BPE 2 (pole IV), and the color turned blue (Out $=$ '1'), as shown in Figure 1a (b). The UV-vis spectra (Supplementary Figure S5) and normalized Abs values of deposited PB $(700 \mathrm{~nm})$ on pole IV (Figure 1b) were in good agreement with the color changes under different inputs. The input and output states produced a truth table (Figure 1c) that meets the characteristic of a 2to-1 encoder. For the construction of the 1-to-2 decoder, the driving voltages with different directions and suitable values/supply time were used as the inputs, the color changes at pole IV (no color change = ' 0 ', turn blue $($ deposited $\mathrm{PB})=$ ' 1 ') and at pole II (no color change $=$ ' 0 ', turn green $\left(\mathrm{ABTS}^{+}\right)={ }^{\prime} 1$ ') were identified as Out $\mathbf{1}$ and Out 2, respectively. In the presence of a negative voltage $(+/-)($ Input $=$ ' 0 '), as discussed with the 2-to-1 encoder, $\mathrm{PB}$ is deposited on the driving cathode, not on pole IV, and no color change was observed at pole IV (Out $\mathbf{1}={ }^{\prime} 0$ '). Meanwhile, the electrochemical oxidation of ABTS in the presence of $\mathrm{H}_{2} \mathrm{O}_{2}$ will occur at the anode of BPE 1 (pole II), and a green color was observed (Out $2=^{\prime} 1$ '), as shown in Figure 1a $\left(a^{\prime}\right)$. After a positive voltage $(-/+)($ Input $=$ ' 1 ') was supplied, the polarity of the two BPEs was reversed compared with the previously mentioned negative voltage. $\mathrm{PB}$ was deposited on the cathode of BPE 2 (pole IV), and a blue color was observed (Out $1=$ ' 1 '). Oxidation of ABTS occurs on the anode of BPE 2 (pole III), not on pole II, and no color change was observed (Out 2='0'). The

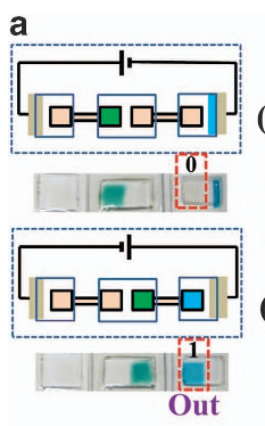

b

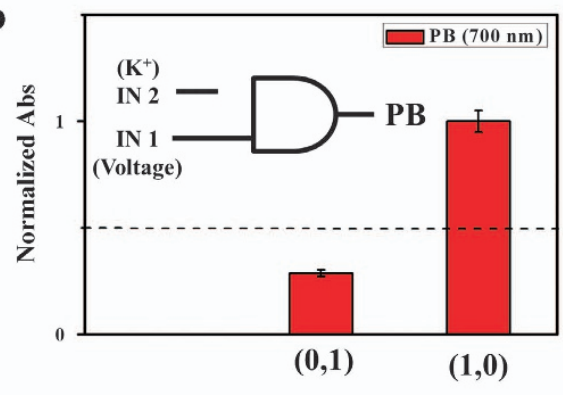

Input Combinations
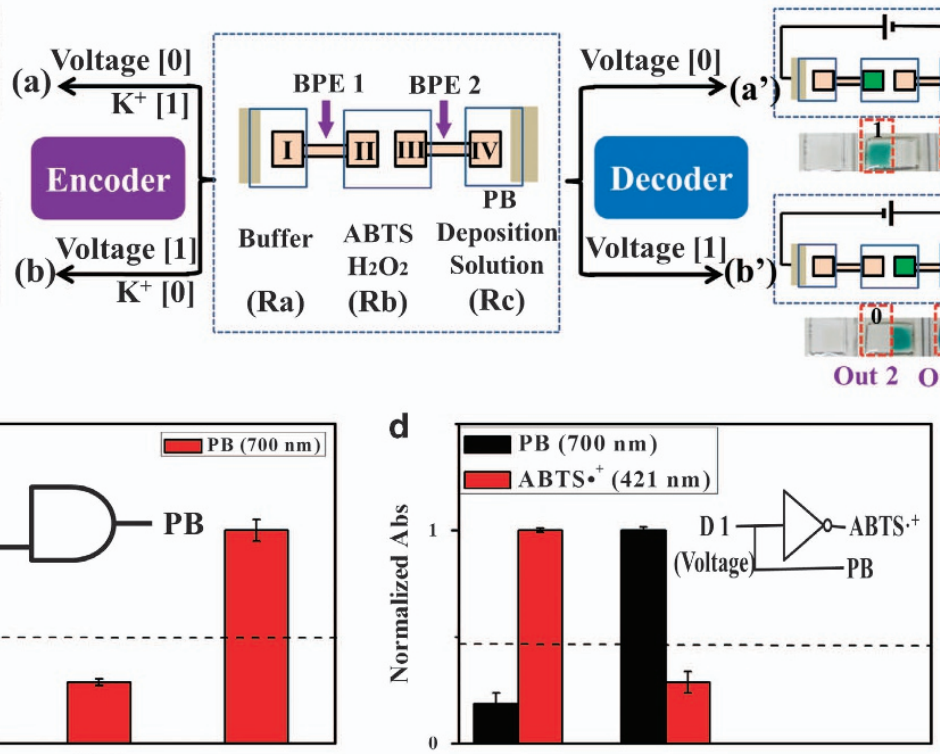

(0)

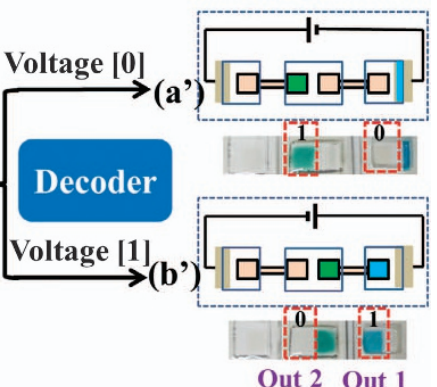

Input Combinations

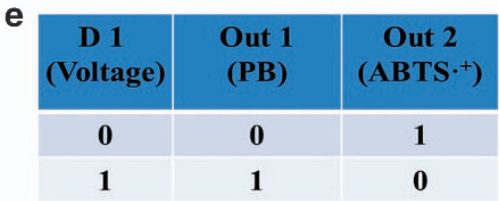

Figure 1 Schematic illustration of the proposed encoder and decoder (a). Column bars of normalized Abs value of PB under different inputs (b), inset is the logic symbol of 2-to-1 encoder; Truth table of the 2-to-1 encoder (c). Column bars of normalized Abs value of deposited PB (black) and ABTS.+ (red) under different inputs (d), inset is the logic symbol of 1-to-2 decoder; Truth table of the 1-to-2 decoder (e). 
a

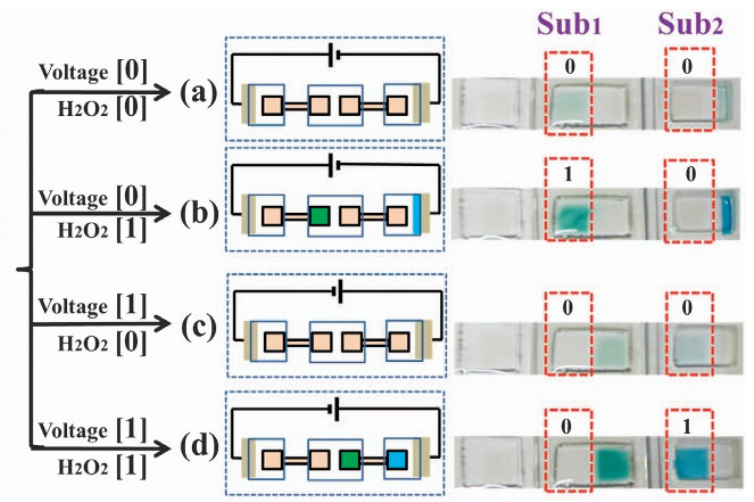

b

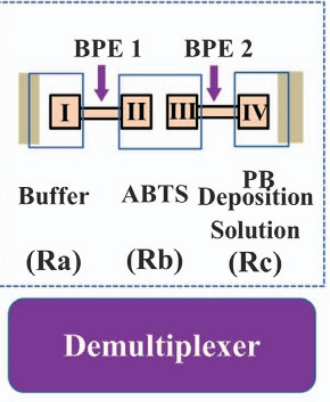

\begin{tabular}{|c|c|c|c|}
\hline $\begin{array}{c}\mathrm{S}_{1} \\
(\text { Voltage })\end{array}$ & $\begin{array}{c}\mathbf{I}_{1} \\
\left(\mathrm{H}_{2} \mathrm{O}_{2}\right)\end{array}$ & $\begin{array}{c}\text { Sub1 } \\
(\text { ABTS-+}\end{array}$ & $\begin{array}{c}\text { Sub2 } \\
(\mathbf{P B})\end{array}$ \\
\hline $\mathbf{0}$ & $\mathbf{0}$ & $\mathbf{0}$ & $\mathbf{0}$ \\
\hline $\mathbf{0}$ & $\mathbf{1}$ & $\mathbf{1}$ & $\mathbf{0}$ \\
\hline $\mathbf{1}$ & $\mathbf{0}$ & $\mathbf{0}$ & $\mathbf{0}$ \\
\hline $\mathbf{1}$ & $\mathbf{1}$ & $\mathbf{0}$ & $\mathbf{1}$ \\
\hline
\end{tabular}

c

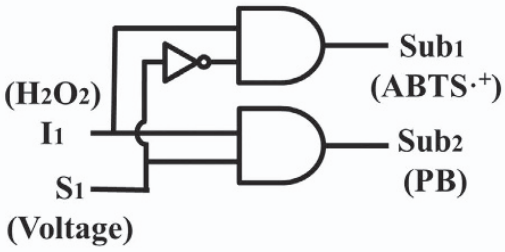

Figure 2 Schematic illustration of the proposed 1-to-2 demultiplexer (a); Truth table (b) and logic symbol (c) of the 1-to-2 demultiplexer.

corresponding UV-vis spectra and normalized Abs results of $\mathrm{PB}$ $(700 \mathrm{~nm})$ and $\mathrm{ABTS}^{+}(421 \mathrm{~nm})$ on the two poles are presented in Supplementary Figure S6 and Figure 1d, respectively. All the above results are presented in the truth table (Figure 1e), which features the characteristics of the 1-to-2 decoder.

\section{Operation of a 1-to-2 demultiplexer}

In addition to the data/code mutual-conversion by the encoders and decoders through information processing, data compression and decompression also play a key role in molecular logic computation. ${ }^{18,42}$ Thus, it is also essential to construct multiplexers and de-multiplexers. ${ }^{43,44}$ To demonstrate the applicability of the platform, a conceptual 1-to-2 demultiplexer was fabricated based on the same BPE platform. 1-to-2 demultiplexer can transmit one input signal to two output channels under the control of a selector. The initial state of the demultiplexer is shown in Figure 2a. The voltage directions were taken as the selector $\left(\mathbf{S}_{1}\right)$, and $\mathrm{H}_{2} \mathrm{O}_{2}$ was used as input $\mathbf{I}_{1}$. The color changes at pole II and pole IV were considered as two outputs: $\mathrm{Sub}_{1}$ and $\mathrm{Sub}_{2}$, respectively. In the absence of $\mathrm{H}_{2} \mathrm{O}_{2}$ and regardless of the voltage directions $\left(\mathbf{S}_{\mathbf{1}} \& \mathbf{I}_{\mathbf{1}}={ }^{\prime} 0,0\right.$ ' or ' 1,0 '), as shown in Figure 2a (a, c), no obvious color change was observed at both pole II and pole IV, resulting in an output of $0\left(\mathrm{Sub}_{1}={ }^{\prime} 0\right.$ ', $\mathrm{Sub}_{2}={ }^{\prime} 0$ '). This result is attributed to the fact that the oxidation of ABTS and deposition of $\mathrm{PB}$ could not occur efficiently without the participation of $\mathrm{H}_{2} \mathrm{O}_{2}$ under insufficient driving voltage $(-4 \mathrm{~V}$ or $+3.8 \mathrm{~V})$. In the presence of a negative voltage $(-4 \mathrm{~V},+/-)$ and $\mathrm{H}_{2} \mathrm{O}_{2}\left(\mathbf{S}_{1} \& \mathbf{I}_{1}={ }^{\prime} 0,1^{\prime}\right)$, the electrochemical oxidation of ABTS occurs at the anode of BPE 1 (pole II), and a green color was observed $\left(\mathbf{S u b}_{1}={ }^{\prime} 1\right.$ '). PB was also deposited on the driving cathode, and no color change was observed at pole IV $\left(\mathrm{Sub}_{2}={ }^{\prime} 0\right.$ ' $)$, as shown in Figure $2 \mathrm{a}(\mathrm{b})$. In the presence of a positive voltage $(+3.8 \mathrm{~V},-/+)$ and $\mathrm{H}_{2} \mathrm{O}_{2}\left(\mathrm{~S}_{1} \& \mathrm{I}_{1}=' 1,1\right.$ '), the ABTS color change occurs on the anode of BPE 2 (pole III), and no change is observed at pole II $\left(\mathbf{S u b}_{1}={ }^{\prime} 0\right.$ '). PB was deposited on the cathode of BPE 2 (pole IV), and a blue color was observed $\left(\mathbf{S u b}_{2}={ }^{\prime} 1\right.$ '). The Abs results under different input combinations (Supplementary Figure S7) were consistent with the corresponding color changes at pole II and pole IV. The corresponding truth table and logic symbol are shown in
Figures $2 \mathrm{~b}$ and $\mathrm{c}$ and show the successful construction of the 1-to-2 demultiplexer. A three-input keypad lock based on the same platform was also constructed and is shown in Supplementary Figure S8.

\section{Operation of a three-input concatenated logic circuit}

In addition to the frequently operated advanced logic devices, complicated concatenated logic circuits, which are usually difficult to fabricate at the molecular scale, connected by several logic devices can perform sophisticated multi-level logic computations. ${ }^{10,14,45}$ Our bipolar electrochemistry-based universal logic platform can not only operate advanced logic devices but also perform concatenated logic computation. A three-input concatenated logic circuit with dual output was subsequently fabricated. Voltage with different directions and suitable values/supply time $(\mathbf{C 1}), \mathrm{H}_{2} \mathrm{O}_{2}(\mathbf{C} 2)$ and $\mathrm{GSH}(\mathbf{C} 3)$ were used as the three inputs. The input definitions were the same as in the above constructed logic devices. For the outputs, the color changes at pole II (no color change $=$ ' 0 ', turn green $\left(\mathrm{ABTS}^{+}\right)={ }^{+}{ }^{\prime} 1$ ') and pole IV (no color change $=$ ' 0 ', turn blue $($ deposited $\mathrm{PB})=$ ' 1 ') were designated output OT 1 and output OT 2, respectively.

The equivalent logic symbol of the three-input concatenated logic circuit shown in Figure $3 \mathrm{c}$ can be illustrated using the states of the two outputs as follows. For output OT 1, as discussed above, the electrochemical oxidation of ABTS can only occur in the presence of a negative voltage and $\mathrm{H}_{2} \mathrm{O}_{2}$ and the absence of GSH $(\mathrm{C} 1$ \& $\mathrm{C} 2$ \& C3 = '0, 1, 0'), producing a green color at pole II (OT 1 = ' 1 '), as shown in Figure $3 \mathrm{a}$ (b). Voltages with different directions and suitable values/supply time and GSH were used as the inputs of the NOR logic gate, and the outputs were different combinations of voltages and GSH. For the output states of the NOR logic gate, only when both of the two inputs were ' 0 ' (negative voltage and without GSH) was the output of the NOR logic gate ' 1 ', and other combinations of voltage and GSH generated output ' 0 '. The different outputs of the NOR logic gate were transmitted as one input of a downstream AND logic gate, whose other input was $\mathrm{H}_{2} \mathrm{O}_{2}$. Thus, a NOR logic gate cascaded by an AND gate (NOR-AND) meets the output states of OT 1. Accompanied by the NOR-AND logic gate achieved at pole II under different inputs, the color changes (PB) at pole IV (OT 2) were also regulated 

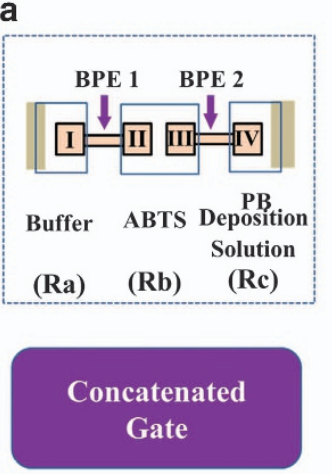

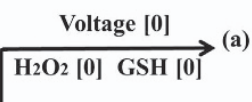
$\mathrm{H}_{2} \mathrm{O}_{2}$ [1] GSH [0]

Voltage [0]

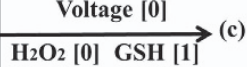

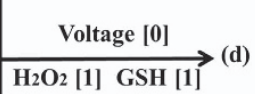

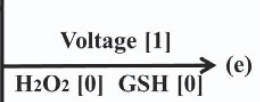
$\underset{\mathrm{H}_{2} \mathrm{O}_{2} \text { [1] GSH [0] }}{\stackrel{\text { Voltage [1] }}{\longrightarrow}}$ (f)
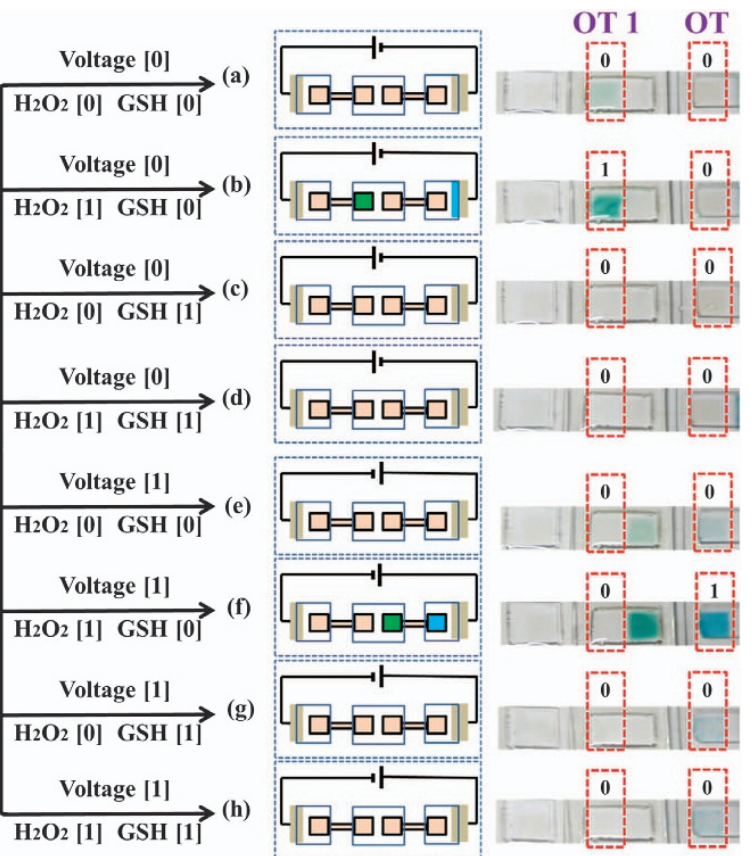

b

\begin{tabular}{|c|c|c|c|c|}
\hline $\begin{array}{c}\mathrm{C} 1 \\
(\text { Voltage })\end{array}$ & $\begin{array}{c}\mathrm{C} 2 \\
\left(\mathrm{H}_{2} \mathrm{O}_{2}\right)\end{array}$ & $\begin{array}{c}\mathrm{C3} \\
(\mathrm{GSH})\end{array}$ & $\begin{array}{c}\text { OT 1 }^{+} \\
\left(\text {ABTS }^{+}\right)\end{array}$ & $\begin{array}{c}\text { OT 2 } \\
(\mathrm{PB})\end{array}$ \\
\hline $\mathbf{0}$ & $\mathbf{0}$ & $\mathbf{0}$ & $\mathbf{0}$ & $\mathbf{0}$ \\
\hline $\mathbf{0}$ & $\mathbf{1}$ & $\mathbf{0}$ & $\mathbf{1}$ & $\mathbf{0}$ \\
\hline $\mathbf{0}$ & $\mathbf{0}$ & $\mathbf{1}$ & $\mathbf{0}$ & $\mathbf{0}$ \\
\hline $\mathbf{0}$ & $\mathbf{1}$ & $\mathbf{1}$ & $\mathbf{0}$ & $\mathbf{0}$ \\
\hline $\mathbf{1}$ & $\mathbf{0}$ & $\mathbf{0}$ & $\mathbf{0}$ & $\mathbf{0}$ \\
\hline $\mathbf{1}$ & $\mathbf{1}$ & $\mathbf{0}$ & $\mathbf{0}$ & $\mathbf{1}$ \\
\hline $\mathbf{1}$ & $\mathbf{0}$ & $\mathbf{1}$ & $\mathbf{0}$ & $\mathbf{0}$ \\
\hline $\mathbf{1}$ & $\mathbf{1}$ & $\mathbf{1}$ & $\mathbf{0}$ & $\mathbf{0}$ \\
\hline
\end{tabular}

C

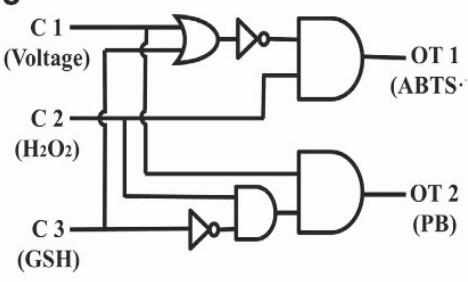

Figure 3 Schematic illustration of the proposed concatenated logic circuit (a), Truth table (b) and logic symbol (c) of the concatenated logic circuit.

by the same three inputs at the same time. For output OT 2, similar to the reactions of ABTS at pole II, only in the presence of a positive voltage and $\mathrm{H}_{2} \mathrm{O}_{2}$ and the absence of GSH $(\mathbf{C} 1 \& \mathbf{C} 2 \& \mathbf{C} 3=$ ' $1,1,0$ ') will $\mathrm{PB}$ be deposited on pole IV with an accompanying blue color (OT $2={ }^{\prime} 1$ '), as shown in Figure $3 \mathrm{a}$ (f). GSH was used as an inhibitory element of $\mathrm{H}_{2} \mathrm{O}_{2}$, and the two reactants composed an INHIBIT logic gate, whose outputs were different combinations of GSH and $\mathrm{H}_{2} \mathrm{O}_{2}$. For the output states of the INHIBIT logic gate, the output was ' 1 ' only in the presence of $\mathrm{H}_{2} \mathrm{O}_{2}$ and absence of $\mathrm{GSH}\left(\mathrm{H}_{2} \mathrm{O}_{2}=\right.$ ' 1 ', $\mathrm{GSH}=$ ' 0 '). All other combinations of $\mathrm{H}_{2} \mathrm{O}_{2}$ and $\mathrm{GSH}$ resulted in output ' 0 '. The different outputs of the INHIBIT logic gate were then transferred as one input of the subsequent AND logic gate, whose other inputs were the voltage directions and suitable values/supply time. The cascade logic device 'INHIBIT-AND' could also feature the output states of OT 2. Two cascade logic devices 'NOR-AND' and 'INHIBIT-AND' could properly represent the output states of OT 1 and OT 2 under different input combinations of the three same inputs. Two devices composed the three-input concatenated logic circuit with dual outputs, as shown in Figures $3 \mathrm{~b}$ and $\mathrm{c}$. The color changes of ABTS and PB under different inputs were easily observed, as shown in Figure 3a, and corresponding Abs spectra are shown in Supplementary Figure S9.

\section{CONCLUSION}

In summary, a universal dual-electrochromic BPE-based logic platform was fabricated for the first time and used to operate various visual advanced logic gates, including an encoder, a decoder, a demultiplexer, a keypad lock and a three-input concatenated logic circuit with two visual outputs. The electrochemical oxidation of ABTS and the electrodeposition of PB were integrated into one closed BPE system, and corresponding color changes at specific poles were used as visual outputs, which are rapidly responsive and can be easily observed by the naked eye by modulating the input combinations. This type of visual color-switch platform provides a prototype for the fabrication of other visual advanced logic devices. We envision that this technology can be miniaturized and operated on one integrated chip driven by a single electric field for lab-on-chip complex data processing, accompanied by further development of the chip technology in the future.

\section{CONFLICT OF INTEREST}

The authors declare no conflict of interest.

\section{ACKNOWLEDGEMENTS}

This work was supported by the National Natural Science Foundation of China (Grant No. 21427811), Youth Innovation Promotion Association CAS 
(No.2016208), MOST China (No. 2016YFA0203200 and 2016YFA0201300) and Jilin province science and technology development plan project 20170101194JC.

Author contributions: QZ and DF contributed equally to this work. QZ made the BPEs and performed the experiments. DF designed the operation of all the logic circuits. QZ, XZ and JL perfected the design of the BPE. JL and EW supervised the project. In addition, all of the authors contributed to the final version of the manuscript.

\section{PUBLISHER'S NOTE}

Springer Nature remains neutral with regard to jurisdictional claims in published maps and institutional affiliations.

1 Willner, I., Shlyahovsky, B., Zayats, M. \& Willner, B. DNAzymes for sensing, nanobiotechnology and logic gate applications. Chem. Soc. Rev. 37, 1153-1165 (2008).

2 Seelig, G., Soloveichik, D., Zhang, D. Y. \& Winfree, E. Enzyme-free nucleic acid logic circuits. Science 314, 1585-1588 (2006).

3 de Silva, A. P. \& Uchiyama, S. Molecular logic and computing. Nat. Nanotechnol. 2, 399-410 (2007).

4 Shlyahovsky, B., Li, Y., Lioubashevski, O., Elbaz, J. \& Willner, I. Logic gates and antisense DNA devices operating on a translator nucleic acid scaffold. ACS Nano $\mathbf{3}$, 1831-1843 (2009).

5 Fan, D., Wang, E. \& Dong, S. A DNA-based parity generator/checker for error detection through data transmission with visual readout and an output-correction function. Chem. Sci. 8, 1888-1895 (2017).

6 Fan, D., Wang, E. \& Dong, S. Simple, fast, label-free, and nanoquencher-free system for operating multivalued DNA logic gates using polythymine templated CuNPs as signal reporters. Nano. Res. 10, 2560-2569 (2017).

7 Fan, D., Zhu, J., Liu, Y., Wang, E. \& Dong, S. Label-free and enzyme-free platform for the construction of advanced DNA logic devices based on the assembly of graphene oxide and DNA-templated AgNCs. Nanoscale 8, 3834-3840 (2016).

8 Fan, D., Wang, K., Zhu, J., Xia, Y., Han, Y., Liu, Y. \& Wang, E. DNA-based visual majority logic gate with one-vote veto function. Chem. Sci. 6, 1973-1978 (2015).

9 Pischel, U. Advanced molecular logic with memory function. Angew. Chem. Int. Ed. 49, 1356-1358 (2010)

10 Feng, L., Lyu, Z., Offenhausser, A. \& Mayer, D. Multi-level logic gate operation based on amplified aptasensor performance. Angew. Chem. Int. Ed. 54, 7693-7697 (2015)

11 Mailloux, S., Gerasimova, Y. V., Guz, N., Kolpashchikov, D. M. \& Katz, E. Bridging the two worlds: a universal interface between enzymatic and DNA computing systems. Angew. Chem. Int. Ed. 54, 6562-6566 (2015).

12 Chen, J., Zhou, S. \& Wen, J. Concatenated logic circuits based on a three-way DNA junction: a keypad-lock security system with visible readout and an automatic reset function. Angew. Chem. Int. Ed. 54, 446-450 (2015).

13 Kang, D., White, R.-J., Xia, F., Zuo, X., Vallée-Bélisle, A. \& Plaxco, K.-W. DNA biomolecular-electronic encoder and decoder devices constructed by multiplex biosensors. NPG Asia Mater. 4, e1 (2012).

14 Jiang, X. J. \& Ng, D. K. Sequential logic operations with a molecular keypad lock with four inputs and dual fluorescence outputs. Angew. Chem. Int. Ed. 53, 10481-10484 (2014).

15 Margulies, D., Melman, G. \& Shanzer, A. A molecular full-adder and full-subtractor, an additional step toward a moleculator. J. Am. Chem. Soc. 128, 4865-4871 (2006).

16 Liu, S., Li, M., Yu, X., Li, C.-Z. \& Liu, H. Biomacromolecular logic gate, encoder/ decoder and keypad lock based on DNA damage with electrochemiluminescence and electrochemical signals as outputs. Chem. Commun. 51, 13185-13188 (2015).

$17 \mathrm{Li}$, H., Liu, Y., Dong, S. \& Wang, E. DNA-based advanced logic circuits for nonarithmetic information processing. NPG Asia Mater. 7, e166 (2015).

18 Orbach, R., Remacle, F., Levine, R. D. \& Willner, I. DNAzyme-based 2:1 and 4:1 multiplexers and 1:2 demultiplexer. Chem. Sci. 5, 1074 (2014).

19 Freeman, R., Finder, T. \& Willner, I. Multiplexed analysis of $\mathrm{Hg} 2+$ and $\mathrm{Ag}+$ ions by nucleic acid functionalized CdSe/ZnS quantum dots and their use for logic gate operations. Angew. Chem. Int. Ed. 48, 7818-7821 (2009).

20 Fan, D., Wang, E. \& Dong, S. Exploiting polydopamine nanospheres to DNA computing: a simple, enzyme-free and G-quadruplex-free dna parity generator/checker for error detection during data transmission. ACS Appl. Mater. Inter. 9, 1322-1330 (2017).

21 Li, H., Hong, W., Dong, S., Liu, Y. \& Wang, E. A resettable and reprogrammable DNAbased security system to identify multiple users with Hierarchy. ACS Nano. 8, 2796-2803 (2014)
22 Jiang, Q., Wang, Z.-G. \& Ding, B. Programmed colorimetric logic devices based on DNAgold nanoparticle interactions. Small 9, 1016-1020 (2013).

$23 \mathrm{Bi}$, S., Yan, Y., Hao, S. \& Zhang, S. Colorimetric logic gates based on supramolecular DNAzyme structures. Angew. Chem. Int. Ed. 49, 4438-4442 (2010).

24 Xianyu, Y., Wang, Z., Sun, J., Wang, X. \& Jiang, X. Colorimetric logic gates through molecular recognition and plasmonic nanoparticles. Small 10, 4833-4838 (2014).

25 Wang, L., Lian, W. \& Liu, H. A resettable keypad lock with visible readout based on closed bipolar electrochemistry and electrochromic poly (3-methylthiophene) films. Chem. Eur. J. 22, 4825-4832 (2016).

26 Liu, D., Chen, W., Sun, K., Deng, K., Zhang, W., Wang, Z. \& Jiang, X. Resettable, multireadout logic gates based on controllably reversible aggregation of gold nanoparticles. Angew. Chem. Int. Ed. 50, 4103-4107 (2011).

27 Poje, J.-E., Kastratovic, T., Macdonald, A.-R., Guillermo, A.-C., Troetti, S.-E., Jabado, O.-J., Fanning, M.-L., Stefanovic, D. \& Macdonald, J. Visual displays that directly interface and provide read-outs of molecular states via molecular graphics processing units. Angew. Chem. Int. Ed. 53, 9222-9225 (2014).

28 Fosdick, S.-E., Knust, K.-N., Scida, K. \& Crooks, R.-M. Bipolar electrochemistry. Angew. Chem. Int. Ed. 52, 10438-10456 (2013).

29 Loget, G., Zigah, D., Bouffier, L., Sojic, N. \& Kuhn, A. Bipolar electrochemistry: from materials science to motion and beyond. Acc. Chem. Res. 46, 2513-2523 (2013).

30 Zhang, X., Zhai, Q., Xing, H., Li, J. \& Wang, E. Bipolar electrodes with $100 \%$ current efficiency for sensors. ACS Sens. 2, 320-326 (2017).

31 Loget, G., Li, G. \& Fabre, B. Logic gates operated by bipolar photoelectrochemical water splitting. Chem. Commun. 51, 11115-11118 (2015).

32 Zhan, W. \& Crooks, R.-M. Microelectrochemical logic circuits. J. Am. Chem. Soc. 125, 9934-9935 (2003).

33 Chang, B.-Y., Crooks, J.-A., Chow, K.-F., Mavré, F. \& Crooks, R.-M. Design and operation of microelectrochemical gates and integrated circuits. J. Am. Chem. Soc. 132, 15404-15409 (2010)

34 Wu, M.-S., Yuan, D.-J., Xu, J.-J. \& Chen, H.-Y. Electrochemiluminescence on bipolar electrodes for visual bioanalysis. Chem. Sci. 4, 1182 (2013).

35 Zhai, Q., Zhang, X., Han, Y., Zhai, J., Li, J. \& Wang, E. A Nanoscale multichannel closed bipolar electrode array for electrochemiluminescence sensing Platform. Anal. Chem. 88, 945-951 (2016).

36 Zhang, X., Li, J., Jia, X., Li, D. \& Wang, E. Full-featured electrochemiluminescence sensing platform based on the multichannel closed bipolar system. Anal. Chem. 86, 5595-5599 (2014).

37 Liu, H. \& Crooks, R.-M. Paper-based electrochemical sensing platform with integral battery and electrochromic read-out. Anal. Chem. 84, 2528-2532 (2012).

38 Zhang, X., Zhang, L., Zhai, Q., Gu, W., Li, J. \& Wang, E. Self-powered bipolar electrochromic electrode arrays for direct displaying applications. Anal. Chem. 88, 2543-2547 (2016)

39 Xiao, Y., Pavlov, V., Niazov, T., Dishon, A., Kotler, M. \& Willner, I. Catalytic beacons for the detection of DNA and telomerase activity. J. Am. Chem. Soc. 126, 7430-7431 (2004)

40 Zhang, H.-R., Wang, Y.-Z., Zhao, W., Xu, J.-J. \& Chen, H.-Y. Visual color-switch electrochemiluminescence biosensing of cancer cell based on multichannel bipolar electrode chip. Anal. Chem. 88, 2884-2890 (2016).

41 de Silva, A. P. Molecular computing - a layer of logic. Nature 454, 417-418 (2008).

42 Szacilowski, K. Digital information processing in molecular systems. Chem. Rev. 108, 3481-3548 (2008).

43 AltanáBozdemir, 0. Proof of principle for a molecular 1: 2 demultiplexer to function as an autonomously switching theranostic device. Chem. Sci. 4, 858-862 (2013).

44 Andreasson, J. \& Pischel, U. Molecules with a sense of logic: a progress report. Chem. Soc. Rev. 44, 1053-1069 (2015).

45 Elbaz, J., Lioubashevski, O., Wang, F., Remacle, F., Levine, R.-D. \& Willner, I. DNA computing circuits using libraries of DNAzyme subunits. Nat. Nanotechnol. 5, 417-422 (2010)

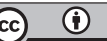

This work is licensed under a Creative Commons Attribution 4.0 International License. The images or other third party material in this article are included in the article's Creative Commons license, unless indicated otherwise in the credit line; if the material is not included under the Creative Commons license, users will need to obtain permission from the license holder to reproduce the material. To view a copy of this license, visit http:// creativecommons.org/licenses/by/4.0/

(C) The Author(s) 2017

Supplementary Information accompanies the paper on the NPG Asia Materials website (http://www.nature.com/am) 